\title{
Editorial
}

\section{Thyroid cancer in the era of endocrine disrupting compounds}

\author{
Cristina Preda \\ Guest Editor
}

Professor, MD, PhD, Department of Endocrinology, "Grigore T. Popa" University of Medicine and Pharmacy, Iași, Romania; "Sf. Spiridon” Emergency County Clinical Hospital, Iași, Romania

Thyroid cancer has the highest prevalence of all endocrine malignancies, accounting for $1-3 \%$ of all human tumors [1]. The incidence of differentiated thyroid cancer has increased globally in the last 10-20 years, faster than that of any other cancer type [2]. The rising incidence has been observed in females, young adults, and children. The possible explanation might be the new achievements in diagnostic procedures, as thyroid ultrasonography and elastography, plus molecular testing and tumoral markers [3, 4]. Even though "over diagnosis" cannot be excluded, the increased incidence for all tumor sizes may suggest that other factors, such as lifestyle changes and environmental influences may contribute to a real increase.

Thyroid cancer is two to four times more frequent in women than in men. It is the second most frequent cancer in the age group 0-49 years, and the fifth most frequent in the age group 50-69 years. Thyroid cancer is also one of the most common tumors for the age group 15-39 years. In children, before the age of 10 , the occurrence of thyroid cancer is very rare [1].

The most frequent histopathological types are papillary and follicular, or welldifferentiated forms of thyroid cancer. They account for $85-90 \%$ and $5-10 \%$ of all thyroid cancers, respectively. Less common forms are medullary carcinoma (2\%), Hurthle cell carcinoma, and the extremely rare and aggressive anaplastic thyroid carcinoma [1]. A combination of tumors in the thyroid it is possible to appear. Cases of synchronous follicular carcinoma, papillary carcinoma, and medullary carcinoma in the same thyroid gland have also been described, but they are very rare. The coexistence of two adjacent but histologically distinct and morphologically independent malignant tumors in the same organ with no histological admixture is called collision tumor. The various types described include the more common papillary carcinoma - medullary carcinoma collision tumors [5].

The cause of thyroid carcinoma is multifactorial and the potential risk factors are both exogenous and endogenous. The wellknown endogenic factors such as age, gender, personal history of benign thyroid disease, family history of thyroid cancer, obesity or insulin resistance are accompanied by exogenic ones like exposure to radiation, iodine intake, diet, and environmental pollutants. All these factors have a potential to promote mutagenesis of thyroid cells. lodine intake is known to influence the histological type of thyroid cancer. lodine deficiency is a risk factor for the occurrence of follicular thyroid cancer, whereas the iodine prophylaxis is associated with an increased risk of papillary thyroid carcinoma [6]. The assessment of patients with papillary carcinoma residing in regions characterized by different levels of iodine, showed that high iodine intake could represent a significant risk factor for the occurrence of BRAF mutations, and the development of papillary thyroid carcinoma. It is possible that iodine deficiency may lead to thyroid cancer through an increase of thyroid stimulating hormone [6]. 
The endocrine disrupting compound is an exogenous substance or mixture that alters function(s) of the endocrine system, and consequently causes adverse health effects in an intact organism, or its progeny or (sub) populations. The source of exposure is cosmetics and personal care products, food contact materials, pharmaceutics, pesticides, cleaning products and more.

Phenols, pesticides, and chlorinated compounds show thyroid disrupting activities. BRAF mutations are likely associated with environmental exposure in chemical carcinogenesis.

The endocrine disrupting compounds may target every step of the thyroid axis: iodine uptake, thyroid hormone synthesis and secretion, thyroid hormone serum protein binding, thyroid hormone metabolism, or thyroid hormone action on receptor level.

Due to the multifactorial etiology of thyroid cancer, synergistic effects among different risk factors and/or different chemicals must be considered. Chronic exposure to a mixture of chemicals with carcinogenic potential can

\section{References}

1. Benedetti $M$, Zona $A$, Contiero $P$, D'Armiento E, lavarone I, Airtum Working Group. Incidence of thyroid cancer in Italian contaminated sites. Int J Environ Res Public Health. 2020; 18(1):191. doi: 10.3390/ijerph18010191

2. Drozd V, Saenko V, Branovan DI, Brown K, Yamashita S, Reiners C. A search for causes of rising incidence of differentiated thyroid cancer in children and adolescents after Chernobyl and Fukushima: Comparison of the clinical features and their relevance for treatment and prognosis. Int $J$ Environ Res Public Health. 2021; 18(7):3444. doi: 10.3390/ijerph18073444

3. Cepeha CM, Paul C, Borlea A, et al. The value of strain elastography in predicting autoimmune thyroiditis. Diagnostics (Basel). 2020; 10(11):874. doi: 10.3390/diagnostics10110874.

4. Ciobanu D, Caruntu ID, Lozneanu L, Andriescu EC, Leustean L, Giusca SE. Tubulin, a possible explain the increase in BRAF mutations associated with thyroid papillary cancer observed worldwide [6].

Another very important aspect is the genetic profile that might confer susceptibility to environmental agents, similar to pharmacogenomic profiles that can predict those most likely to respond to a given drug. Studies linking specific polymorphisms in genes involved in thyroid hormone synthesis, secretion, metabolism, and action with indices of thyroid function following exposure to putative toxicants are necessary to determine the validity of this concept [7].

Epidemiological studies through advanced design with individual exposure assessment are necessary, using human biomonitoring data and pharmacokinetic models, in order to investigate the disrupting effect of chemical compounds. It is mandatory to study the cofactors of exposure to compounds with endocrine disrupting properties. Combined data from animal models are required to clarify the exact role of endocrine disruptors in humans [6].

marker for the prognostic stratification and therapy in papillary thyroid carcinoma. Farmacia. 2018; 66(4):635-643. doi: 10.31925/farmacia.2018.4.12

5. Li H, Livneh $N$, Dogan S, Shaha AR: The growth kinetics of collision nodal metastasis from medullary and papillary thyroid carcinomas: a case report. Eur Thyroid J. 2021; 10(4):345-352. doi: 10.1159/000511184

6. Gorini F, lervasi G, Coi A, Pitto L, Bianchi F. The role of polybrominated diphenyl ethers in thyroid carcinogenesis: is it a weak hypothesis or a hidden reality? From facts to new perspectives. Int $J$ Environ Res Public Health. 2018; 15(9):1834. doi: 10.3390/ijerph15091834

7. Brent GA, Braverman LE, Zoeller RT. Thyroid health and the environment. Thyroid. 2007; 17(9):807-809. doi: 10.1089/thy.2007.1514 